\title{
Western Diet Deregulates Bile Acid Homeostasis, Cell Proliferation, and Tumorigenesis in Colon
}

\section{Dermadi, Denis}

2017-06-15

Dermadi , D , Valo , S , Ollila , S, Soliymani , R, Sipari , N, Pussila , M , Sarantaus , L , Linden , J , Baumann , M \& Nystrom , M 2017 , ' Western Diet Deregulates Bile Acid Homeostasis, Cell Proliferation, and Tumorigenesis in Colon ' , Cancer Research , vol. 77 , no. 12 , pp. 3352-3363 . https://doi.org/10.1158/0008-5472.CAN-16-2860

http://hdl.handle.net/10138/237004

https://doi.org/10.1158/0008-5472.CAN-16-2860

unspecified

publishedVersion

Downloaded from Helda, University of Helsinki institutional repository.

This is an electronic reprint of the original article.

This reprint may differ from the original in pagination and typographic detail.

Please cite the original version. 


\title{
Western Diet Deregulates Bile Acid Homeostasis, Cell Proliferation, and Tumorigenesis in Colon
}

\author{
Denis Dermadi ${ }^{1,2,3}$, Satu Valo, ${ }^{1,4}$, Saara Ollila ${ }^{5}$, Rabah Soliymani ${ }^{6}$, Nina Sipari ${ }^{7}$, \\ Marjaana Pussila', Laura Sarantaus', Jere Linden ${ }^{8}$, Marc Baumann ${ }^{6}$, and \\ Minna Nyström ${ }^{\top}$
}

\section{Abstract}

Western-style diets (WD) high in fat and scarce in fiber and vitamin D increase risks of colorectal cancer. Here, we performed a long-term diet study in mice to follow tumorigenesis and characterize structural and metabolic changes in colon mucosa associated with WD and predisposition to colorectal cancer. WD increased colon tumor numbers, and mucosa proteomic analysis indicated severe deregulation of intracellular bile acid (BA) homeostasis and activation of cell proliferation. WD also in- creased crypt depth and colon cell proliferation. Despite increased luminal BA, colonocytes from WD-fed mice exhibited decreased expression of the BA transporters FABP6, OST $\beta$, and ASBT and decreased concentrations of secondary BA deoxycholic acid and lithocholic acid, indicating reduced activity of the nuclear BA receptor FXR. Overall, our results suggest that WD increases cancer risk by FXR inactivation, leading to BA deregulation and increased colon cell proliferation. Cancer Res; 77(12); 3352-63. @2017 AACR.

\section{Introduction}

Western-style diet (WD), rich in energy derived from saturated fats of animal origin and scarce of fiber, vitamin D, calcium, and folic acid, is recognized as one of the main risk factors for colorectal cancer (1). Dietary fat has been implicated to increase colorectal cancer risk, and associations have been found between colorectal cancer incidence, saturated fat intake, and high consumption of red and processed meat (2).

WD has been shown to promote important oncogenic processes in colon tissue, aberrant apoptosis (3), proinflammatory stimulus (4), and increased neoplastic growth (5). Our previous study highlighted that WD elevates fatty acid metabolism, thus providing a source of reactive oxygen species (ROS) in colon tissue (6). Consumption of high-fat diet like (HFD) WD

'Department of Biosciences, Genetics, University of Helsinki, Helsinki, Finland. ${ }^{2}$ Laboratory of Immunology and Vascular Biology, Department of Pathology, Stanford University School of Medicine, Stanford, California. ${ }^{3}$ The Center for Molecular Biology and Medicine, Veterans Affairs Palo Alto Health Care System, Palo Alto, California. ${ }^{4}$ Department of Medical and Clinical Genetics, University of Helsinki, Helsinki, Finland. ${ }^{5}$ Research Program Unit, Faculty of Medicine, University of Helsinki, Helsinki, Finland. ${ }^{6}$ Meilahti Clinical Proteomics Core Facility, Department of Biochemistry and Developmental Biology, Medicum, University of Helsinki, Helsinki, Finland. ${ }^{7}$ Viikki Metabolomics Unit, Department of Biosciences, University of Helsinki, Helsinki, Finland. ${ }^{8}$ Department of Basic Veterinary Sciences, University of Helsinki, Helsinki, Finland.

Note: Supplementary data for this article are available at Cancer Research Online (http://cancerres.aacrjournals.org/).

Corresponding Authors: Denis Dermadi, Stanford University, Miranda Av. 3801 Palo Alto, CA 94304. Phone: 415-794-3999; E-mail: ddermadi@stanford.edu or Minna Nyström, Department of Biosciences, Genetics, University of Helsinki, FI-00014 Helsinki, Finland. E-mail: minna.nystrom@helsinki.fi

doi: 10.1158/0008-5472.CAN-16-2860

(C)2017 American Association for Cancer Research.
(20\%-35\% fat) results in significantly higher excretion of fecal secondary bile acids (BA), mainly deoxycholic acid (DCA) and lithocholic acid (LCA; ref. 7). BAs are known for emulsifying effect on dietary fat; high secretion of DCA in colon lumen and high BA concentrations in feces are indeed correlated with increased incidence of colorectal cancer $(8,9)$. Furthermore, high concentrations of luminal BAs are associated with increased proliferation of colon epithelia (10) also indicating pathogenicity. Overall, BA homeostasis seems to be a critical tumor suppressor in colon $(11,12)$.

In the present study, we characterized functional changes induced by Western-style diet in the colon mucosa of C57BL/ 6 mice. We complemented two high-throughput proteomics methods, 2D difference gel electrophoresis (DIGE) and liquid chromatography coupled mass spectrometry (LC-MS), both of which provide insights into final cellular changes, unlikely to be determined with other methods such as RNA sequencing. Our proteomic analysis indicated aberrance in proliferation, apoptosis, and BA metabolism in WD-fed mice. Concurrent increase of proliferation and avoidance of apoptosis in normal tissue is the most important oncogenic driver event when considering hallmarks of cancer. Previous studies on WD effects in mice have associated WD with hyperproliferation due to reduced amount of dietary calcium (3) without addressing known effects of BAs on proliferation. Previous studies have linked colorectal cancer with high BA concentrations in colon lumen and feces (13). Here, we studied colon crypt depth, cell proliferation, and measured BA concentrations both in colon lumen and in colon tissue, because only intracellular BAs can directly affect nuclear receptors, gene expression, and modulate cellular processes. Our comprehensive proteomic study and targeted metabolomics coupled with histologic analysis of colon tissue show that Western-style diet severely activates cell proliferation and causes malabsorption of BAs in colon epithelia, two phenomena associated with intestinal tumorigenesis. 


\section{Materials and Methods}

\section{Mice and diets}

C57BL/6 mice were bred and treated according to the study protocol approved by the National Animal Experiment Board in Finland (ESLH-2008-06502/Ym-23).

Mice were randomly divided into two dietary groups at the age of 6 weeks and fed ad libitum with control diet AIN-93G (American Institute for Nutrition, AIN; ref. 14) or modified AIN diet to mimic human Western style diet (Supplementary Table S1; Harlan Teklad) previously described (6). Mice were kept in 12-hour light/dark cycle with controlled temperature and humidity.

Sexes were equally represented in all experimental groups. We sacrificed mice and collected specimens at 6 weeks, 12, 18, and 21 months of age. Size of each experimental group was between 8 and 12 animals. Experimental groups were 6-week, 12-month AIN, 12-month WD, 18-month AIN, 18-month WD, 21-month AIN, and 21-month WD. Histologic analyses of neoplasias/ tumors were carried out at The Finnish Centre for Laboratory Animal Pathology, University of Helsinki, Finland. Detailed description of mice used in each analysis and tumor information is presented in Supplementary Table S2.

\section{Specimens}

A longitudinal piece of proximal colon mucosa was separated from the smooth muscle layer under a dissecting microscope. Samples for protein extractions were rinsed with tris-based buffer, snap frozen, and stored at $-80^{\circ} \mathrm{C}$. Samples for RNA extraction were stored in RNAlater (Qiagen) at $-80^{\circ} \mathrm{C}$ until extraction. Proximal colon tissue for BA analysis was stored in optimal cutting temperature reagent at $-80^{\circ} \mathrm{C}$.

\section{Protein extraction}

Colon mucosa was mechanically homogenized in $(10-15 \mu \mathrm{L}$ lysis buffer/mg tissue) 2D Protein Extraction Buffer-VI (GE Healthcare), pH 8.5 Tris (30 mmol/L; Sigma-Aldrich), Protease Inhibitor Mix (1/100; GE Healthcare), and phosphatase inhibitor cocktail 2 and 3 (Sigma-Aldrich). Homogenate was vigorously shaken for 20 minutes at $4{ }^{\circ} \mathrm{C}$ followed by centrifugation at $15,000 \mathrm{~g}$ for 20 minutes. Supernatant was collected, snap frozen, and stored at $-80^{\circ} \mathrm{C}$. The concentration of total protein extracts was measured with 2D Quant Kit (GE Healthcare).

\section{D-DIGE}

We used 2D-DIGE to detect the most prominent changes in the colon proteome expression, as described previously (6). The reagents, instruments, and software were manufactured by GE Healthcare if not stated otherwise. Samples were labeled with Cy3 and Cy5 (400 pmol of dye for $50 \mu \mathrm{g}$ of sample) according to the manufacturer's instructions. An internal standard was created by pooling $25 \mu \mathrm{g}$ of each protein sample and labeled with Cy2.

Equal amounts $(50 \mu \mathrm{g})$ of internal standard (Cy2), Cy3, and Cy5 labeled samples were actively introduced into immobilized pH 3-11 nonlinear gradient (IPG) strips $24 \mathrm{~cm}$ long, using the Ettan IPGphor II unit. After protein separation, according to their isoelectric point, IPG strips were equilibrated with $1 \%$ dithiothreitol (DTT) and 2.5\% iodoacetamide. Finally, proteins were separated in second dimension using precast large $12.5 \%$ SDSPAGE gels with constant power of $15 \mathrm{~W}$ per gel. Gels were scanned with Typhoon Trio scanner and images acquired with excitation/ emission values 480/530 nm (Cy2), 520/590 nm (Cy3), and 620/ $680 \mathrm{~nm}$ (Cy5). Maximum intensities between different channels differed less than $20 \%$ to $30 \%$. DeCyder 2D 7.0 was used for analysis of gel images.

\section{Protein identification}

In order to excise proteins from the 2D gels, we silver-stained them with a PlusOne Silver staining kit (GE Healthcare). Proteins were in-gel digested with trypsin (Trypsin gold; Promega) as previously described (6). MALDI-MS and MS/MS analyses were performed with UltrFlexTreme (Bruker Daltonics) using SmartBeam II $355 \mathrm{~nm}$ laser. Calibration was externally performed with peptide calibration standard (Bruker Daltonics). MALDI-MS and MS/MS spectra were acquired by accumulation of 5,000 to 7,000 or 10,000 to 20,000 shots, respectively. The spectra were searched against UniProt/SwissProt database [release of 2013_12, (541954 sequences; 192668437 residues), taxon: Mus musculus (16649 sequences), www.uniprot.org] using Mascot server (Matrix Science, www.matrixscience.com), FlexAnalysis, and BioTools software (Bruker Daltonics). We used the following search parameters: cleaving enzyme trypsin, one missed cleavage allowed, peptide mass tolerance \pm 0.1 Da for MS searches, and fragment tolerance $\pm 0.5- \pm 1.5 \mathrm{Da}$ for combined MS/MS searches. Variable and fixed modifications were oxidized methionine and carbamidomethylation of cysteine, respectively.

\section{Sample preparation for LC-MS}

Results obtained with 2D-DIGE were validated with nonlabeling quantitative LC-MS. We combined equal amounts $(10 \mu \mathrm{g})$ of total protein extract of individual mouse belonging to each study group and from created pools used $10 \mu \mathrm{g}$ for digestion, using modified filter-aided sample preparation protocol (15). Briefly, the lysis buffer was exchanged by washing protein extracts several times with $8 \mathrm{~mol} / \mathrm{L}$ urea, $0.1 \mathrm{~mol} / \mathrm{L}$ Tris, $\mathrm{pH} 8$ (urea buffer, UB). Reduction with $5 \mathrm{mmol} / \mathrm{L}$ DTT and alkylation with $50 \mathrm{mmol} / \mathrm{L}$ iodoacetamide in UB were followed by digestion with 1:50 w/v of Lysine-C endopeptidase (Wako) in $4 \mathrm{~mol} / \mathrm{L}$ urea/0.1 mol/L Tris $\mathrm{pH} 8$ at room temperature overnight. The peptide digests were collected by centrifugation, and trypsin solution was added in a ratio of $1: 50 \mathrm{w} / \mathrm{v}$ in $50 \mathrm{mmol} / \mathrm{L}$ ammonium bicarbonate. As before, the digests were collected and combined. The peptide samples were cleaned using C18 - reverse phase ZipTip (Millipore), resuspended in $1 \%$ trifluoroacetic acid and ultrasonicated in water bath for 1 minute.

\section{LC-MS}

We analyzed triplicates of each pooled sample. Digested proteins (200 ng) were injected for LC-MS analysis. The peptides were separated by nanoAcquity ultra pressure liquid chromatography (UPLC) system (Waters) equipped with a trapping column $5 \mu \mathrm{m}$ Symmetry C18 $180 \mu \mathrm{m} \times 20 \mathrm{~mm} \mathrm{C18}$ reverse phase (Waters), followed by an analytical $1.7 \mu \mathrm{m}, 75 \mu \mathrm{m} \times 250 \mathrm{~mm}$ BEH-130 C18 reversed-phase column (Waters), in a single pump trapping mode. The injected sample analytes were trapped at a flow rate of $15 \mu \mathrm{L} / \mathrm{min}$ in $99.5 \%$ of solution A ( $0.1 \%$ formic acid). After trapping, the peptides were separated with a linear gradient of $3 \%$ to $35 \%$ of solution B ( $0.1 \%$ formic acid/acetonitrile), for 90 minutes at a flow rate $0.3 \mu \mathrm{L} / \mathrm{min}$, and stable column temperature of $35^{\circ} \mathrm{C}$. Every run was followed by two empty runs to wash out any remaining peptides from previous runs. The samples were run in data-independent analysis mode (MS), in a Synapt G2-S mass spectrometer (Waters), by alternating between low collision 
energy (6V) and high collision energy ramp in the transfer compartment (20 to $45 \mathrm{~V}$ ) and using 1-second cycle time. The separated peptides were detected online with mass spectrometer, operated in positive, resolution mode in the range of $\mathrm{m} / \mathrm{z} 50$ $2,000 \mathrm{amu}$. Note that $150 \mathrm{fmol} / \mu \mathrm{L}$ of human $\left[\mathrm{Glu}^{1}\right]$-fibrinopeptide B (Sigma-Aldrich) in 50\% acetonitrile $/ 0.1 \%$ formic acid solution at a flow rate of $0.3 \mu \mathrm{L} / \mathrm{min}$ was used for a lock mass correction applied every 30 seconds.

\section{Quantitation and database search}

Relative quantification between pooled samples using precursor ion intensities was performed with TransOmics Informatics for Proteomics software (Nonlinear Dynamics, Waters) and ProteinLynx Global Server (PLGS V3.0). We used following MS parameters: low energy threshold of 135 counts, elevated energy threshold of 30 counts, and intensity threshold of precursor/ fragment ion cluster 750 counts. Chromatograms were automatically aligned by the TransOmics software using the default values. Those that had alignment score $\geq 70 \%$ to the reference run were selected for further analysis. Database searches were carried out against UniProtKB-SwissProt (release 2014_04, taxon: Mus musculus, 32538 entries) with Ion Accounting algorithm and using automatic peptide and fragment tolerance, maximum protein mass: $500 \mathrm{kDa}$, minimum fragment ions matches per protein $\geq 7$, minimum fragment ions matches per peptide $\geq 3$, minimum peptide matches per protein $\geq 1$, primary digest reagent: trypsin, missed cleavages allowed: 2, fixed modification: carbamidomethylation C, variable modifications: phosphorylation of serine, tyrosine and threonine residues, oxidation of methionine, and FDR $<4 \%$. All identifications were subsequently refined to Mus musculus only identifiers and the protein lists simplified by protein grouping. Protein quantitation was performed entirely on nonconflicting protein identifications, using precursor ion intensity data and standardized expression profiles.

\section{Western blot analyses}

Antibodies against FABP6 (1:1,000, 12579-RP09; Sino Biological Inc.), ASBT (1:1,000, SLC10A2, CPBT-46249RM; Creative Diagnostics), FXR (NR1H4, 1:500, A9033A; Novus Biologicals), GAPDH $(1: 5,000,14 \mathrm{C} 10$; Cell Signaling Technology), and $\alpha$-tubulin (1:20,000, clone DM1A; Sigma-Aldrich) were used in Western blot analyses. Secondary antibodies were IRDye $800 \mathrm{CW}$ goat anti-rabbit IgG (926-32211) and IRDye 680 RD goat antimouse IgG (926-68070; LI-COR). Briefly, 10 to $20 \mu \mathrm{g}$ of sample pools containing equal amounts of total protein extracts of each mouse belonging to a respective group were separated on $4 \%$ to $20 \%$ gradient gels (Bio-Rad) and blotted on either Immobilon $\mathrm{P}^{\mathrm{SQ}}$ (Millipore) or Trans-BlotTurbo (Bio-Rad). After antibody incubation, the blots were scanned with Odyssey (LI-COR) and quantified with the Image Studio Lite (LI-COR) software. Loading control, either $\alpha$-tubulin or GAPDH, was used to normalize protein abundance between different experimental groups.

\section{Analysis of bile acids}

BAs were extracted from proximal colon mucosa and fecal content of colon lumen into ethanol as described previously (16). Briefly, lyophilizated samples were pulverized in TissueLyser (Qiagen). Nordeoxycholic acid (NDCA; Steraloids) was added $(1 \mu \mathrm{g})$ to the samples prior the extraction as an internal standard to monitor sample quality and quantify concentration of BA in the samples. Freeze-dried proximal colon mucosa samples were grinded in a ball mill and ultrasonicated (level 9, $120 \mathrm{~W}$, USC600-THD, VWR) in ethanol for 1 hour, heated at $60^{\circ} \mathrm{C}$ for 30 minutes, cooled to the room temperature, and boiled at $90^{\circ} \mathrm{C}$ for 3 minutes. Extracts were centrifuged at 1,600 $\mathrm{g}$ for 10 minutes and supernatant was collected. Ethanol was added to precipitates and vigorously mixed for 1 minute. Supernatant was collected after centrifuging at $11,200 \mathrm{~g}$ for 1 minute. This was repeated twice. Supernatants were pooled and ethanol evaporated in vacuum concentrator. Extracts were dissolved in methanol and purified with HLB cartridges (Waters). Liquid was evaporated and BAs were finally reconstituted in $50 \mu \mathrm{L}$ of methanol and analyzed with UPLC-MS immediately. BAs from feces were extracted with isopropanol without SPE extraction due to the fact that feces contain mostly water and water-soluble inorganic salts, organic material but also small amounts of bacterial matrix and proteins. Liquid-liquid extraction was performed with isopropanol (IPA) to extract BAs and to purify samples from water-soluble content without time consuming SPE $(17,18)$. Samples were extracted twice with $1 \mathrm{~mL}$ IPA, centrifuged, and evaporated to dryness. Dry samples were reconstituted in $200 \mu \mathrm{L}$ of acetonitrile and analyzed with UPLC-MS immediately.

\section{Q-TOF-MS detection of BAs}

Extracted BAs were separated on Acquity UPLC BEH C18 column $(1.7 \mu \mathrm{m}, 50 \times 2.1 \mathrm{~mm}$, Waters $)$ in $+40^{\circ} \mathrm{C}$ attached to Waters Acquity UPLC system (Waters). The mobile phase consisted of (A) $\mathrm{H}_{2} \mathrm{O}$ and (B) acetonitrile (Chromasolv grade; Sigma-Aldrich) both containing $10 \mathrm{mmol} / \mathrm{L}$ ammonium acetate (Fluka/Sigma-Aldrich). A linear gradient started at $95 \%$ of A, decreased to $60 \%$ in 10 minutes, then to $20 \%$ in 24 minutes, then back to $95 \%$ in 24.1 minutes, and left to equilibrate for 1 minute. The injection volume was $3 \mu \mathrm{L}$, and flow rate of the mobile phase was $0.6 \mathrm{~mL} / \mathrm{min}$. UPLC system was interfaced with Waters Synapt G2 HDMS mass spectrometer (Waters). Ionization was performed using an electrospray ionization (ESI) source. Samples were analyzed in negative sensitivity ion mode. Mass range was set from 50 to $600 \mathrm{amu}$. The ionization parameters were optimized with the internal standard, NDCA. BAs were identified by their $\mathrm{m} / \mathrm{z}$ ratio and retention time and by running BA standards deoxycholic acid, cholic acid, hyodeoxycholic acid, chenodeoxycholic acid, ursodeoxycholic acid, LCA, isoLCA, $\alpha$-muricholic acid ( $\alpha \mathrm{MCA}), \beta \mathrm{MCA}, \omega \mathrm{MCA}, \alpha$-tauromuricholic acid ( $\alpha \mathrm{TMCA}$ ), and $\omega \mathrm{TMCA}$ (Steraloids). The chromatograms and mass spectra were analyzed with Waters MassLynx Software (Waters). Unknown metabolites without standard were tentatively identified by their exact mass, relative retention time, and previous publications (19).

\section{Histologic analyses of colon crypts}

We measured the length of five colon crypts per animal and counted the number of cells in each measured crypt in blinded manner (Supplementary Table S2). Hematoxylin and eosin (H\&E)-stained colon tissue sections were visualized by cellSens Dimensions camera (Olympus) mounted on BX63 microscope (Olympus). All measurements were performed using Aperio ImageScope v12.1.0.5029 (Aperio Technologies Inc.). Proliferation index (Ki67 index) was studied by immunohistochemistry. Colon tissue sections were deparaffinized and antigen retrieved with hot citrate buffer, $\mathrm{pH}$ 6. Endogenous peroxidase was blocked with $\mathrm{H}_{2} \mathrm{O}_{2}$ treatment. Tissue sections were incubated overnight with Ki67 antibody $(1: 1,000$, ab15580; 
Abcam) and detected with anti-rabbit polymer (Biocare) and DAB enzymatic system. Slides were scanned with Pannoramic 250 Flash II (3D Histech); visualization and analysis were done in CaseViever (3D Histech). We analyzed 5 crypts per animal (Supplementary Table S2) and calculated a proliferation index dividing number of Ki67-positive cells with the number of total cells within a crypt.

\section{RNA expression studies}

Reverse transcription of mRNA (500 ng) was done with Superscript III (Life Technologies) from individual mice according to the manufacturer's instructions. Quantitative RT-qPCR of genes involved in regulation of BA sensing and metabolism, Fxr (F: 5'-GCAGAGCGTACTCCTCCTGA-3' and R: 5'-GACATGCAGACCTGTTGGAA-3') and Shp (F: 5'-AAGGGCTTGCTGGACAGTTA-3' and R: 5'-TCTCTTCTTCCGCCCTATCA-3'), was studied using SyberGreen chemistry (Thermo Fisher). Individual mice samples (12 and 18 months) were assayed in triplicates for the target genes using the following cycling parameters: 1 cycle of $95^{\circ} \mathrm{C}$ for 10 minutes, 40 cycles of $95^{\circ} \mathrm{C}$ for 15 seconds, and $60^{\circ} \mathrm{C}$ for 1 minute. $\beta$-Actin was used as an endogenous reference gene (F: 5'-CTAAGGCCAACCGTGAAAAG-3' and R: 5'-ACCAGAGGCATACAGGGACA-3'). Thermal cycling and fluorescence data acquisition were performed with the CFX384 Real Time System (Bio-Rad), and Cq values were obtained using the CFX manager Software (Bio-Rad). The mRNA expression changes were analyzed using the comparative $C_{t}\left(\Delta \Delta C_{t}\right)$ method, which presents the data as fold changes in gene expression normalized to the endogenous reference gene and compared with the expressions in the control group (12-month AIN).

\section{Statistical analysis}

The significance of changes detected in mouse weight, length of colon crypts, number of cells, proliferation index, and BA concentrations was analyzed with the Mann-Whitney $U$ test (20).

The mean and median permutation tests (21) were used to analyze the expression data from Western blot and RT-qPCR studies. Pearson correlation was used to analyze expressions obtained from 2D-DIGE, LC-MS, and Western blot studies for selected proteins.

Neoplasias and tumors in general follow negative binomial distribution (22), which was used to analyze our data set. Calculations were performed in $\mathrm{R}$ version 3.2.0. The threshold for significance was $P \leq 0.05$.

The expression changes in 2D-DIGE analysis were detected using the DeCyder 2D version 7.0 (GE Healthcare), and the significance in expression differences between the mouse groups was tested with the Student $t$ test with significance threshold $P \leq 0.01$. We compared WD groups with their control groups (AIN) in order to detect diet effect at different time points. For combined effect of diet and aging, we compared diet groups from different time points. Principal component analysis (PCA) of normalized protein abundances from DeCyder 2D was performed in $\mathrm{R}$ version 3.2.0.

For LC-MS proteomic analysis, we utilized the betweensubject ANOVA design scheme of TransOmics software. The thresholds to accept proteins based on differential intensities between treatment and control groups were absolute fold-change $\geq 2$ computed from averaged and normalized protein intensities, and $P$ value $\leq 0.05$ for ANOVA in all comparisons.

\section{Network analysis}

The lists of up/downregulated protein changes from 2D-DIGE and LC-MS analyses were analyzed with Ingenuity Pathway Analysis (IPA; Qiagen). We used core analysis with following settings: ingenuity knowledge base (genes and endogenous chemicals) with direct and indirect relationships, for network interaction: include endogenous chemicals, 35 molecules per network, and 25 networks per analysis. All data sources were used with confidence: experimentally observed and highly predicted. We selected data obtained in all species with relaxed filter and excluded data on mutant variants. Analyses were exported, and heat maps for upstream regulators, diseases, and functions and classical pathways were created in R package NMF using significant z-scores calculated in IPA.

\section{Results}

Western diet increases weight and induces neoplastic changes in colon

Mice fed with WD showed a trend of increased weight gain (Supplementary Fig. S1A). Male mice showed a significant $(P=$ $0.036)$ difference in average weights between 12-month AIN $(41.41 \mathrm{~g})$ and 12 -month WD (46.50 g) groups, whereas in females, the weight difference was smaller, $30.10 \mathrm{~g}$ and $32.90 \mathrm{~g}$ in 12-month AIN and 12-month WD groups, respectively. At 18 months of age, the average weights of males fed with WD and AIN were $53.91 \mathrm{~g}$ and $42.93 \mathrm{~g}(P=0.019)$ and of females $38.10 \mathrm{~g}$ and $36.55 \mathrm{~g}$, respectively. Interestingly, mice fed with AIN continued to gain weight until 21 months of age, whereas WD-fed mice started to lose their weights after 18 months most probably caused by deterioration of wellbeing in the WD groups.

Altogether 6 adenocarcinomas, 1 adenoma, and 13 hyperplastic foci had developed during the diet experiment, and 16 of them in WD-fed mice (Supplementary Table S2). We built a linear regression model to study the number of tumors in relation to different diets (AIN or WD) and aging $(12,18$, and 21 months). Aging and diet had significant effect on the number of tumors. Coefficients for aging and WD were $1.91, P=0.012$ and $1.41, P=$ 0.012 , respectively. WD on its own increased the likelihood of developing tumors 4 times and aging from 12 to 21 months 6.75 times. Our model allows us to conclude that at 18 months of age, every third mouse fed with WD, and at 21 month of age, each WD fed mouse would develop at least one macroscopic tumor (lower limit: 0.58, upper limit: 1.87; Supplementary Fig. S1B).

\section{Proliferation, apoptosis, and BA transport networks are affected by Western diet}

Increased number of tumors in aging mice is a result of many accumulated alterations occurring in colon epithelium during the feeding experiment. Since proteomes of mice at 21 months did not differ significantly from proteomes of mice at 18 months within the same diet groups (Fig. 1A), we focused on differences in proteomes detected at 12 and 18 months to address changes that may precede and induce tumors in WD mice. To assess the changes in colon mucosa proteome during the feeding experiment, we isolated altogether 77 proximal colon epithelia samples, operated from the Western and control (AIN) diet groups at different time points $(N=10-12$ mice per data point, Supplementary Table S2). Protein lysates of the colon epithelia were analyzed with 2D DIGE (Supplementary Table S2). Altogether, 1,974 proteins were detected, and 
Dermadi et al.
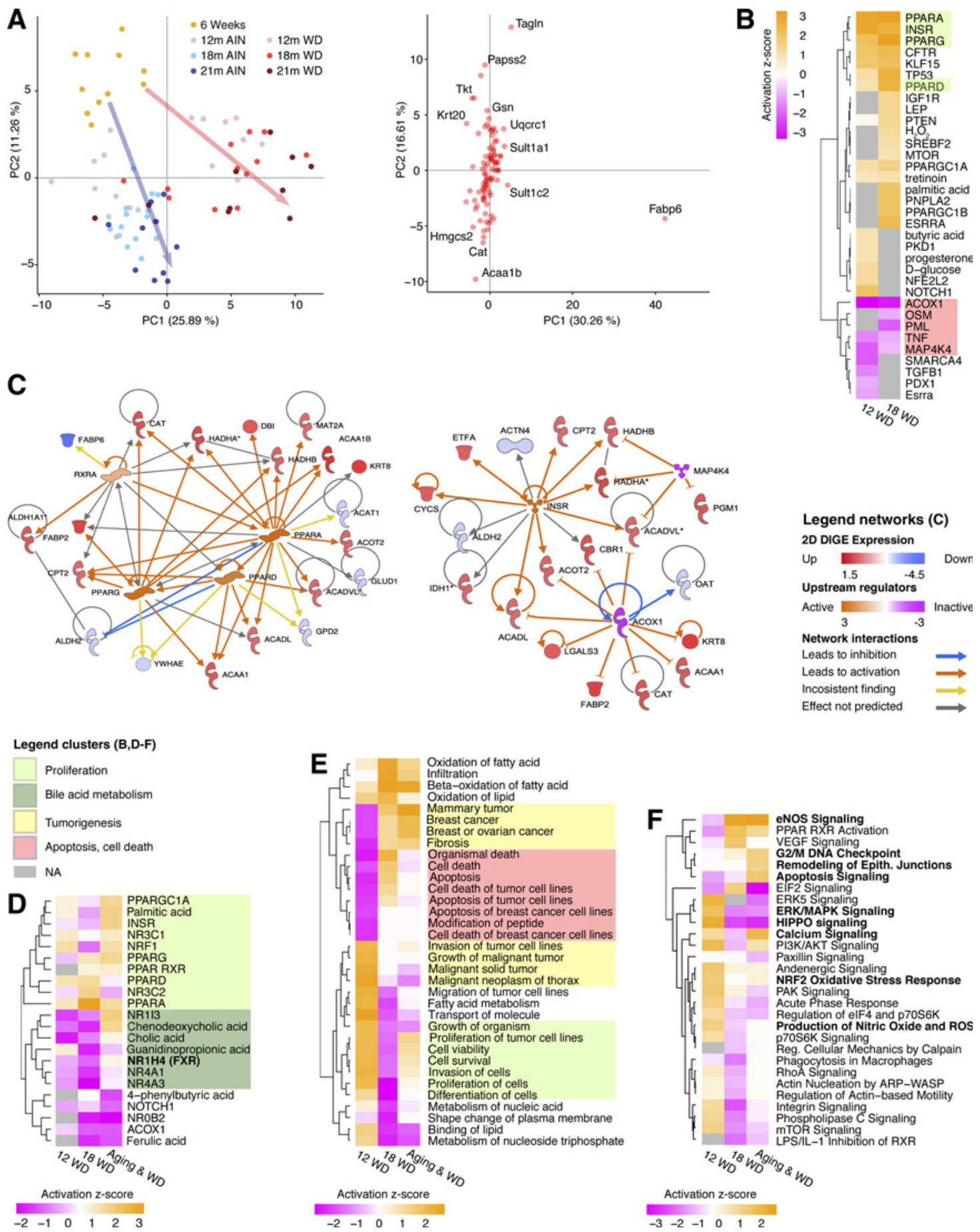

Figure 1.

Systems biology (networks, upstream activators, biological functions, and canonical pathways) analysis of proteomic changes. A, PCA of all mice in diet experiment. Arrows, shift of groups fed with AIN and WD from the mice at experiment start point. PCA of all identified proteins by 2D-DIGE shows proteins that contribute to difference between experimental groups. B, IPA of proteins identified by 2D-DIGE in association with WD revealed activation of genes involved in proliferation (green) and inactivation of genes involved in apoptosis (red) in 12-month WD and 18-month WD. C, Network analysis of proteins identified by 2D-DIGE strongly demonstrates activation of PPARs and INSR and inactivation of ACOX1 and MAP4K4 networks. D, IPA of proteins detected by LC-MS demonstrates activation of proliferation regulators (light green), especially when combining the effect of WD and aging; the same analysis revealed strong inactivation of NR1H4 (FXR) and BAs (dark green). E, Biological functions and diseases based on proteins detected by LC-MS indicate activation of proliferation (green), tumorigenesis (yellow), and deregulation of apoptosis (red) in 12-month WD mice. F, Canonical pathways based on proteins detected by LC-MS supported previous analysis and additionally pointed to active oxidative stress via ROS, metabolic reprogramming, and inflammatory effects of WD consumption. 
approximately $10 \%$ of those showed significant changes between diet groups. We used peptide mass fingerprinting (MALDI-TOF TOF) to identify 96 proteins (Supplementary Table S3), of which 48, 35, and 28 unique proteins showed significant difference in expression when comparing mice fed with AIN and WD at 12, 18, and 21 months of age, respectively (Supplementary Table S4A-S4E). Using these data, the PCA showed that WD-fed mice diverge from AIN-fed mice, and especially they differ from mice at experimental start point (6 weeks) before mouse groups had diet differences. Principal component (PC)1 mainly associated with diet and described up to $26 \%$ variability between individuals, whereas PC2 described additional $11 \%$ of variability and associated mainly with age. Moreover, we utilized PCA to determine proteins with highest variability between different experimental groups. Here, we identified catalase (CAT), cytochrome b-c1 complex subunit 1, mitochondrial (UQCRC1), fatty acid binding protein 6 (FABP6), gelsolin (GSN), 3'-phosphoadenosine 5'phosphosulfate synthase 2 (PAPSS2), sulfotransferase family, cytosolic 1A, phenol-preferring, member 1 (SULT1A1), sulfotransferase family, cytosolic 1C, member 2 (SULT1C2), and transgelin (TAGLN; Fig. 1A).

We further used IPA tool for systems biology approach to identify upstream activators and biological functions enriched in our dataset. IPA analysis revealed peroxisome proliferatoractivated receptor (PPAR) $\alpha, \gamma$, and $\delta$, and insulin receptor as the most prominently activated networks and inactivation of acylCoA oxidase 1, palmitoyl (ACOX1), mitogen-activated protein kinase kinase kinase kinase 4 (MAP4K4), and TNF networks, all pointing to increased proliferation, deregulation of apoptosis, and cell death in colon mucosa exposed to WD (Fig. 1B and C). Highly enriched biological functions for PPAR $\alpha, \gamma$, and $\delta$ were malignant solid tumors (23 proteins, $P=5.44 \mathrm{e}-5$ ), abdominal cancer (19 proteins, $P=8.33 \mathrm{e}-4)$, epithelial cancer (19 proteins, $P=9.11 \mathrm{e}-4)$, breast or colorectal cancer (15 proteins, $P=$ $1.74 \mathrm{e}-4)$ as well as indicated expected malfunctions in fatty acid metabolism (Supplementary Table S5A). Top biological functions associated with ACOX1, insulin receptor (INSR), and MAP4K4 networks were malignant solid tumors (22 proteins, $P=8.84 \mathrm{e}-6)$ and tumorigenesis of tissue (19 proteins, $P=$ 1.84e-4; Supplementary Table S4B). Both large networks, PPARs and ACOX1-INSR-MAP4K4, had in common biological function of inflammation of organ, respectively (Supplementary Table S5A and S5B).

We validated our 2D-DIGE findings by LC-MS (Supplementary Table S6) to rule out biased detection due to inherent limitations of proteomic methods (23). A proportion of approximately $68 \%$ of proteins identified by 2D-DIGE were successfully detected with LC-MS (Supplementary Fig. S2), indicating reliability of the obtained results. Proteomic data analysis with LC-MS provided additional protein expression changes. IPA analysis confirmed the findings of the most significantly altered networks. Cross-comparison analysis of LC-MS proteomic data sets between age groups of 12 and 18 months showed inactivation of FXR (NR1H4) and confirmed previously found changes in PPARs and ACOX1. IPA of biological functions indicated strong inactivation of apoptosis at 12 months in mice fed with WD, together with activation of tumorigenesis and proliferation. In addition, pathway analysis pinpointed to divergent behavior of the same pathways at different time points favoring proliferation, tumorigenesis, and increased concentration of ROS (Fig. 1D-F).

\section{Western diet increases cell proliferation in colon crypts}

To confirm the results of network and functional analyses of proteomic data that suggested increased proliferation together with decreased apoptosis, we measured depth and counted cell number of colon crypts (Fig. 2A and B). Crypts of mice fed with WD were $24 \mu \mathrm{m}$ longer than crypts of control mice at 12 months of age $(P=3.15 \mathrm{e}-9)$ and $24 \mu \mathrm{m}$ longer at 18 months of age $(P=1.32 \mathrm{e}-8)$, respectively. Difference in depth of crypts between diet groups at 21 months was not significant. Contrary to WD mice, crypts of AIN mice expressed continuous growth; $15 \mu \mathrm{m}$ both between 12 and 18 months $(P=9.05 e-5)$ and between 18 and 21 months $(P=5.84 \mathrm{e}-05)$. We observed an increase in the number of cells within crypts of WD mice (Supplementary Fig. S3A) with the highest number of cells in the 12-month WD group. Significantly increased depth of the crypts, higher number of colonocytes, and IPA results in WD mice encouraged us to investigate the effects of $\mathrm{WD}$ on the proliferation zone of colon crypts (Fig. 2C and D). Proliferation index, defined as a ratio between Ki67-positive cells and total cells within the crypt, was $40 \%$ higher in crypts of 12 -month WD mice compared with 12 -month AIN mice $(P=3.228 \mathrm{e}-6)$. Control AIN diet did not cause any significant change in proliferation between different time points, indicating that significant increase in depth of crypts observed between 12month AIN mice and 18-month AIN mice was mainly due to increased cell size and not due to increased cell number (Supplementary Fig. S3B)

\section{WD-challenged colon mucosa reduces BA reabsorption and decreases activity of FXR}

Data from our proteomic analysis suggested that WD leads to disruption of lipid metabolism and detoxification of BAs caused by inactivation of BA sensing and transport (Fig. 1AD). FABP6 transports BAs into nucleus where BAs activate FXR, subsequently regulating expression of genes involved in BA transport (Asbt, Fabp6, Ost $\alpha$, and Ost $\beta$ ), and an epigenetic modifier (Shp). FABP6, an intracellular BA transporter in intestinal epithelial cells, showed the most significant change in expression between different experimental groups in 2D-DIGE (Fig. 3A). We validated the WD-induced reduction of FABP6 protein level with LC-MS and Western blot analyses, and found that the three independent methods showed high correlation (Pearson's coefficient $\geq 0.95, P<0.05$; Supplementary Fig. S4A and S4B). Remarkable WD-induced reduction of FABPG level was observed at 12 months $(-4.52$, $P=1.81 \mathrm{e}-7)$.

With LC-MS, we also detected a significant expression reduction of organic solute transporter $\beta$ subunit (OST $\beta$ ), known as solute carrier family 51, beta subunit (SLC51B), which is exclusively expressed within the basal membrane of colon epithelium and responsible for transport of BAs into extracellular space. OST $\beta$ showed similar expression pattern as FABP6, with significant reduction in expression at 12 months of age $(-2.16, P=0.018$; Fig. 3B)

ASBT, known as solute carrier family 10 member 2 (SLC10A2), is the main BA importer located on the apical membrane of colon cells. Because we were not able to detect this protein in either 2D-DIGE or LC-MS, we studied the expression of this protein by Western blot analysis and found that ASBT was indeed decreased $(-1.57, P=0.018)$ in 12 -month WD mice when compared with mice on control diet (Fig. 3C). 

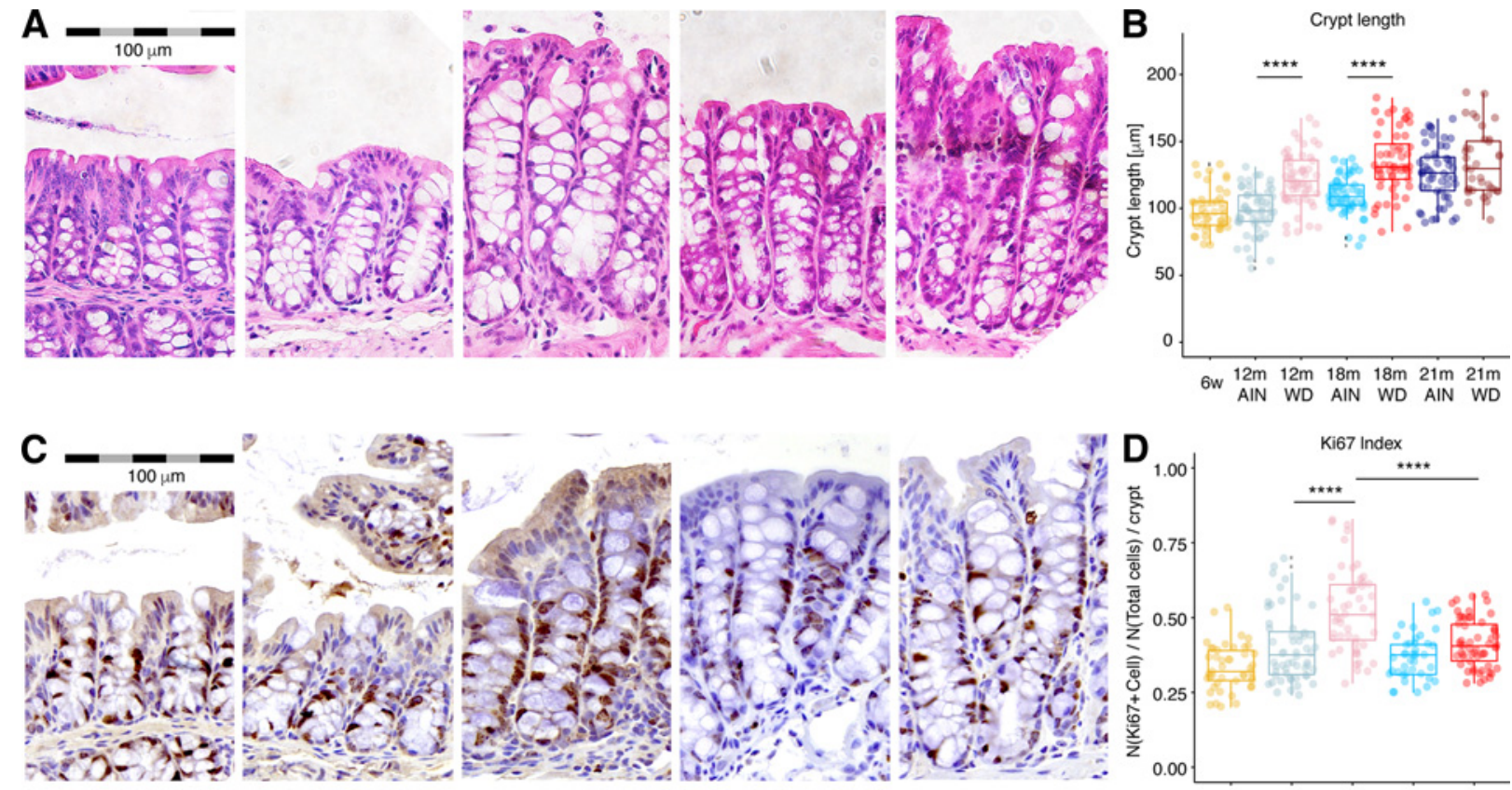

6 Weeks

12 Months AIN

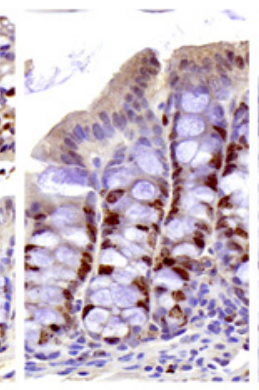

12 Months WD
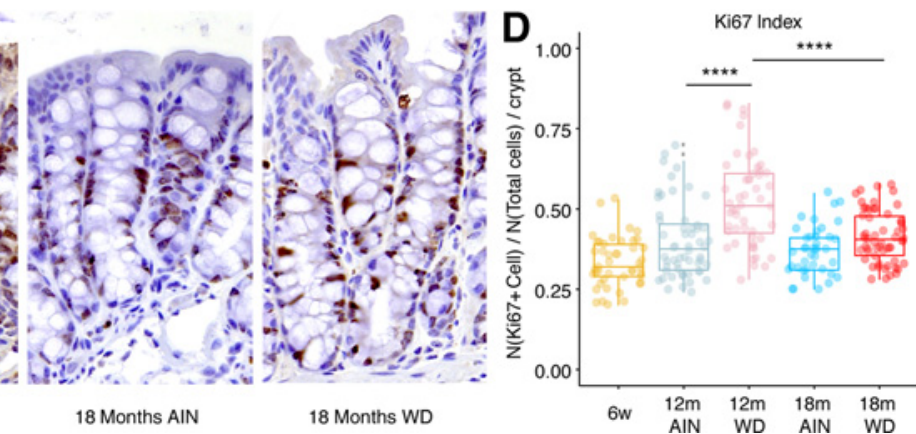

Figure 2.

Analysis of colon crypt depth and proliferation index. A, Representative images of H\&E-stained colon crypts in different experimental groups show significant increase of depth of colon crypts in mice fed with WD. B, Significant increase of crypt depth in 12-month WD and 18-month WD when compared with control groups. C, Representative images of Ki67 proliferation marker show extended proliferation zone in colon crypts of 12-month WD mice. D,

Quantitation of proliferation index. Proliferation zone in colon crypts of 12-month WD mice is significantly extended when compared with controls and with 18-month WD groups. $N=5$ crypts/each mouse; ${ }^{* * * *}, P<0.0001$.

Systems biology analysis suggested inactivation of FXR (Fig. 1D), and reduced expressions of FABP6 and OST $\beta$ strongly supported inactivation of FXR, main nuclear receptor for BAs, either (i) by reduced expression or (ii) by reduced concentration of BA in the tissue. In order to confirm the inactivation of FXR, we analyzed FXR expression by qPCR and Western blot. No significant differences were observed in the
Fxr gene expression or protein abundance when comparing diet groups at 12 months (Fig. 3D; Supplementary Fig. S4C). Although with increased age, FXR expression was upregulated in AIN group (Western blot: $1.4, P=0.004$; qPCR: $1.65, P=$ $0.033)$, the gene and protein expressions of FXR were decreased in 18-month WD mice in comparison with 18 month AIN mice (Western blot: $-1.35, P=0.003$, qPCR:
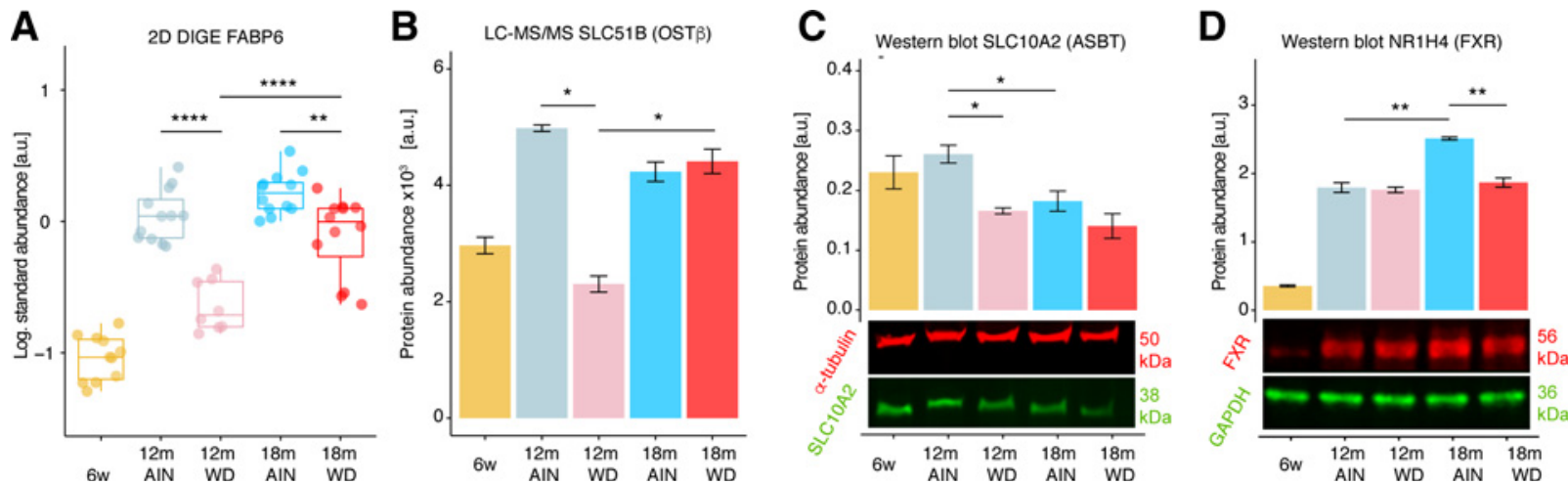

Figure 3.

Changes in BA transport pathway. A, Abundance of FABP6 measured with 2D-DIGE shows remarkable changes between different experimental groups. B, Significant reduction of OST $\beta$ abundance in 12-month WD mice and increase in 18-month WD mice measured with LC-MS. C, Western blot of ASBT shows significantly lower ASBT abundance in 12-month WD. D, Protein abundance of FXR measured with Western blot. Western blots were performed in three independent assays. Error bars, SEM. * $P<0.05$; ${ }^{* *}, P<0.01 ;{ }^{* *}, P<0.001$; and ${ }^{* * *}, P<0.0001$. 

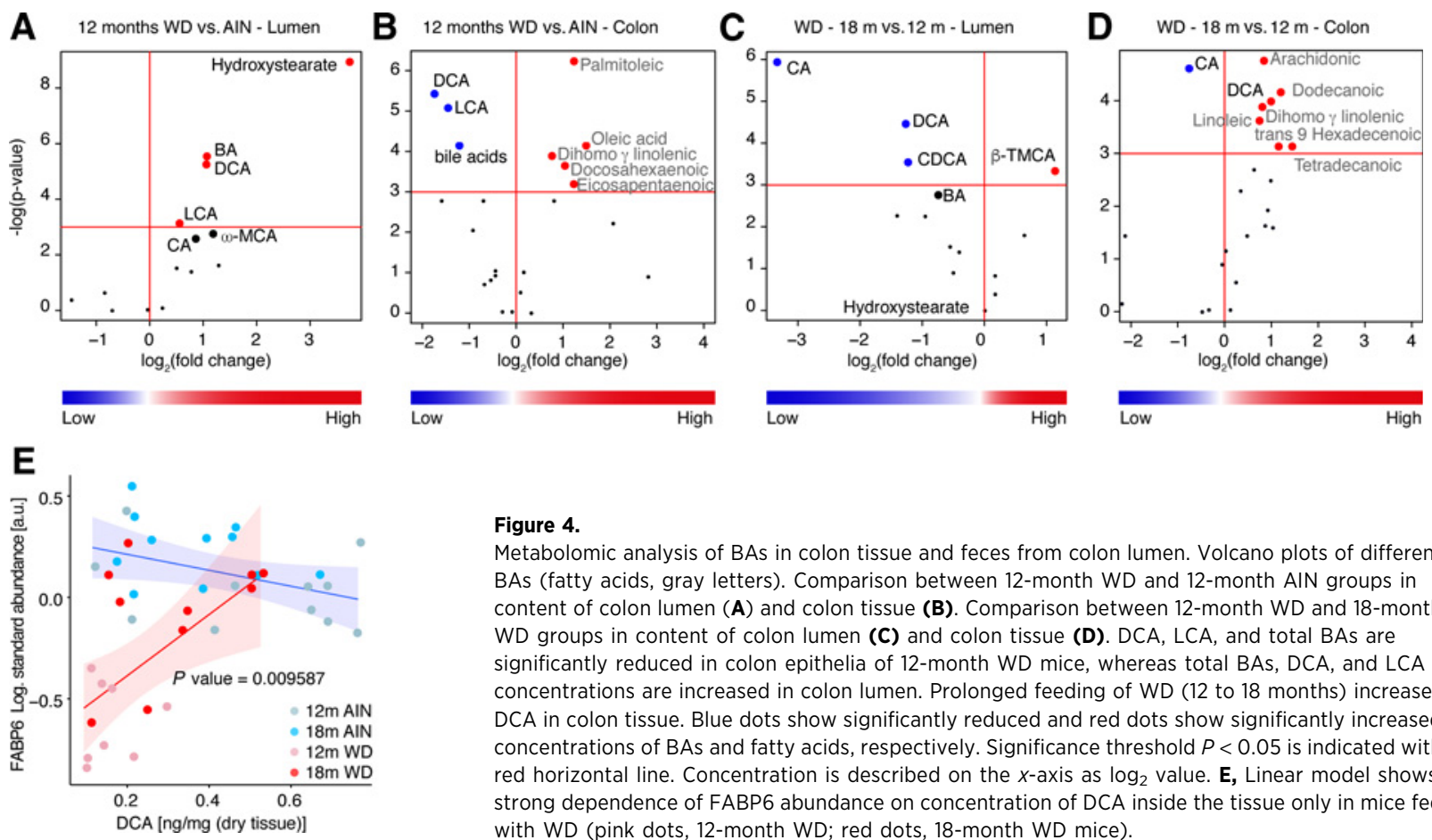

\begin{abstract}
Figure 4.
Metabolomic analysis of BAs in colon tissue and feces from colon lumen. Volcano plots of different BAs (fatty acids, gray letters). Comparison between 12-month WD and 12-month AIN groups in content of colon lumen (A) and colon tissue (B). Comparison between 12-month WD and 18-month WD groups in content of colon lumen (C) and colon tissue (D). DCA, LCA, and total BAs are significantly reduced in colon epithelia of 12-month WD mice, whereas total BAs, DCA, and LCA concentrations are increased in colon lumen. Prolonged feeding of WD (12 to 18 months) increases DCA in colon tissue. Blue dots show significantly reduced and red dots show significantly increased concentrations of BAs and fatty acids, respectively. Significance threshold $P<0.05$ is indicated with red horizontal line. Concentration is described on the $x$-axis as $\log _{2}$ value. $\mathbf{E}$, Linear model shows strong dependence of FABP6 abundance on concentration of DCA inside the tissue only in mice fed with WD (pink dots, 12-month WD; red dots, 18-month WD mice).
\end{abstract}

$-1.79, P=0.006)$. Moreover, Shp, another FXR target protein, showed strongly decreased expression in WD mice when compared with 12-month AIN and 18-month AIN mice (-3.34, $P=0.031$ and $-3.15, P=0.019$ ), respectively (Supplementary Fig. S4D).

No differences in FXR expression were detected at 12 months. We hypothesized that differences in intracellular BA pools could affect FXR activity and mediate target gene transcription; thus, we conducted UPLC-Q-TOF-MS analysis of BAs in colon tissue and feces collected from colon lumen. Interestingly, although concentration of BAs was increased in the luminal feces of 12-month WD mice, indicating elevated $\mathrm{BA}$ excretion (Fig. 4A), the intracellular concentrations of several BAs, DCA, and LCA in particular were significantly reduced in 12-month WD (Fig. 4B), suggesting decreased transport. Concentration of intracellular DCA increased, and the fecal concentration of BAs, mainly DCA and CDCA, decreased over time in mice fed with WD (12-month WD vs. 18-month WD; Fig. 4C and D). No differences were observed in intracellular BA concentrations when comparing 18-month WD with 18-month AIN (Supplementary Fig. S5A). Aging on control diet did not change concentration of BAs in colonocytes (12-month AIN vs. 18-month AIN; Supplementary Fig. S5B). Only luminal tauro-muricholic acids (TMC) were elevated in 18-month WD mice when compared with 18-month AIN; however, aging on control diet reduced concentration of TMCs (Supplementary Fig. S5C and S5D). Relevance of concentration of DCA inside the colon tissue was emphasized in a linear regression analysis, which showed a strong $(P=0.009587)$ association between DCA concentration and abundance of FABP6 in mice fed with WD (Fig. 4E).

\section{Discussion}

WD significantly increased the number of tumors in mouse colon supporting observations from previous studies $(5,24)$. Our main finding is that WD increases cell proliferation and tumorigenesis in colon in association with severe deregulation of intracellular BA homeostasis. Aging increased likelihood of developing tumor, fortifying already established connection between cancer, aging, and lifestyle (25).

We also detected an increased colon crypt depth and cell proliferation in WD-fed mice supporting a recently published result of increased crypt depth caused by HFD (26), which suggests that HFD enhances stemness and tumorigenicity of intestinal progenitors via activation of PPAR $\delta$. We demonstrate that WD results in higher abundance of proteins regulated by PPARs (Fig. 1C-E). Although Beyaz and colleagues focused on changes in intestinal progenitor cells caused by HFD ( $60 \%$ fat content) and we studied the effects of WD ( $40 \%$ fat content) in normal colon mucosa cells, an unsupervised clustering and a heatmap of our 2D-DIGE findings and their RNA-Seq data (GEO: GSE67324) showed a remarkable correlation between the results (Supplementary Fig. S6).

By using 2D-DIGE and MALDI-TOF/TOF, we identified 96 proteins (Supplementary Table S4A-S4E), which successfully defined experimental groups in PCA segregating WD-fed mice from AIN-fed and 6-week-old mice. PCA analysis discriminated proteins (CAT, FABP6, GSN, PAPSS2, SULT1A1, SULT1C2, TAGLN, and UQCRC1) that showed the most significant differences between experimental groups (Fig. 1A). The increased expression of CAT (27) and the decreased expression of UQCRC1 indicate that WD induces oxidative stress and mitochondrial dysfunction $(4,6,28)$. The decreased expression of 
transformation and shape-changing sensitive actin cross-linking/gelling protein, transgelin (TAGLN), has been previously detected in primary colorectal cancer tumors, and reduction of TAGLN has shown to induce proliferation in colon cancer cell lines (29). Similarly, in our study, colon crypts of 12-month WD mice showed increased proliferation. Moreover, PAPSS2 and SULT1A1 are essential in the detoxification of BAs and necessary for avoidance of pathologic conditions such as colon cancer (30). Downregulation of GSN has been observed in many transformed cell lines and tumor tissues (breast, colon, ovaries, prostate, and bladder; ref. 31).

The intracellular BA transporter, FABP6, showed the most significant difference in expression between experimental groups and expressed remarkably lower abundance in mice fed with WD. FABP6 is mainly expressed in the distal portion of the small intestine and in proximal colon, has a high affinity for binding BAs, and is essential for their enterohepatic circulation (32). Expression of Fabp6 is directly induced in a BA-dependent manner by FXR, which is a key regulator of BA homeostasis (33). FABP6 is known to functionally associate with FXR and ASBT in the nucleus and on the membrane, respectively, and to stimulate FXR activity through a mutual interaction augmented by BA uptake (34). Lower abundance of FABP6 indicated that colon tissue of mice fed with WD might have hampered intestinal apical-basal BA transport as seen in Fabp $6^{-1-}$ mice (35). Systems biology analysis of proteomics data suggested activation of
PPARs and inactivation of FXR (NR1H4) networks, indicating disruption of lipid metabolism as well as BA sensing and transport. Moreover, ASBT and OST $\beta$ were downregulated in 12-month WD mice, further suggesting inactive BA transport. Most BAs are efficiently absorbed into the intestinal cells by ASBT, transported in cytosol by FABP6, and exported across the basolateral membrane into the portal circulation by the heterodimeric transporter OST $\alpha /$ OST $\beta$ (Fig. 5A; refs. 33, 36).

The consistent downregulation of BA transporters in 12-month WD mice strongly supported two alternative conditions: (i) lower abundance of FXR, which is a common regulator of BA-related genes (Fabp6, Ost $\beta$, Shp; refs. 33, 3638 ) or (ii) reduced FXR activity due to a lower concentration of BAs. The latter possibility was predicted with IPA $(39,40)$. Interestingly, no significant differences were observed in FXR expression between diet groups at 12 months. Consistent with reduced expression of transporters, LC-MS analysis of BAs revealed a significant decrease in intracellular concentrations of DCA and LCA in the colon epithelium. In contrast, concentrations of fecal BAs were increased in 12-month WD mice reflecting elevated synthesis/excretion and supporting previous studies that demonstrate increased fecal BAs among individuals with high dietary fat intake (7). Furthermore, our linear model showed that the FABP6 abundance is dependent on the intracellular DCA concentration in mice fed with WD. Taken together, the results strongly suggest downregulation of
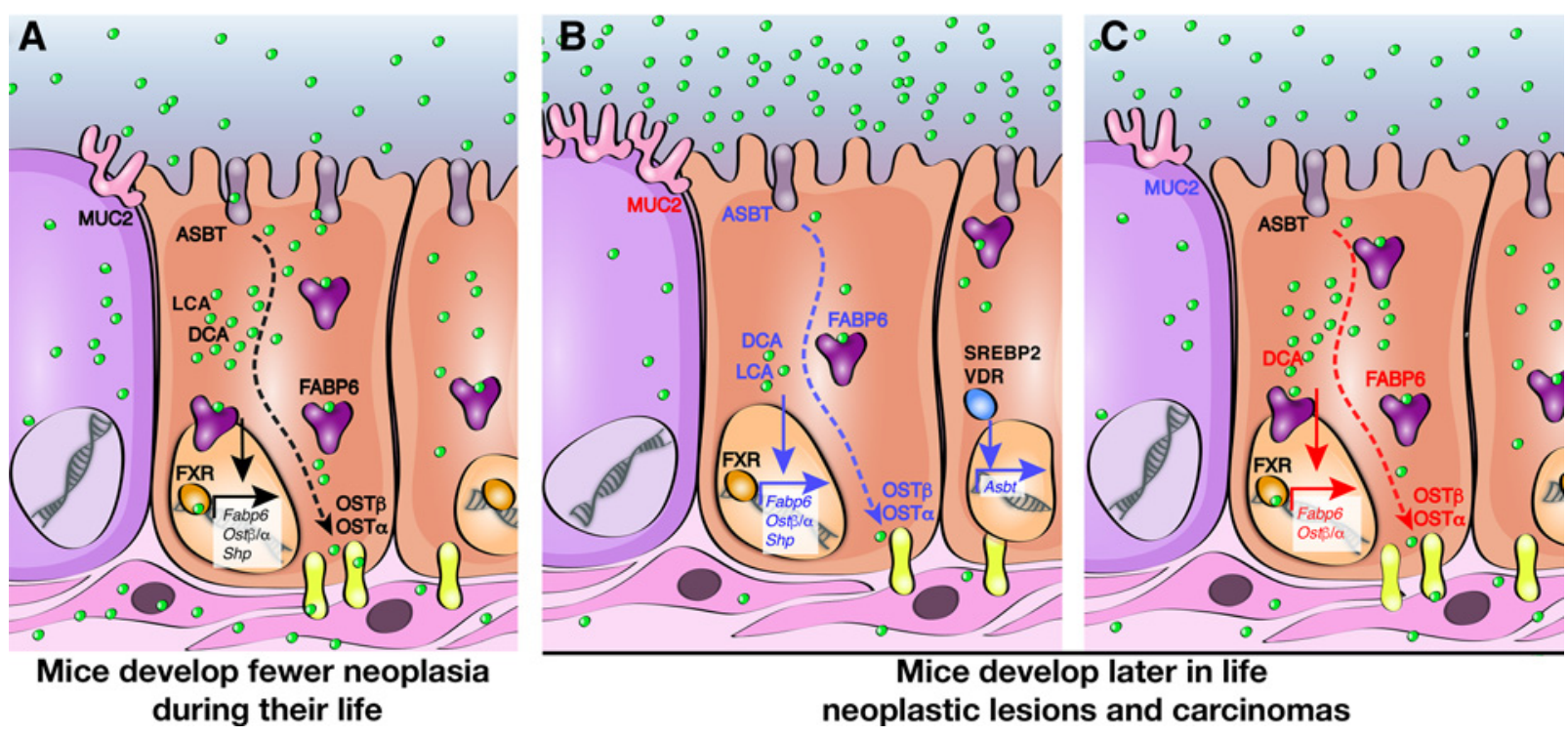

Mice develop later in life neoplastic lesions and carcinomas

Figure 5.

Proposed model: Western diet impairs BA transport in colon and reduces FXR activity. A, Steady state in colonocyte. BAs (green circles) are transported into the colonocyte by ASBT and distributed by FABP6 to FXR and to OST $\alpha /$ OST $\beta$ transporter on the basal membrane for active reuptake. BAs act as activating ligands of FXR, which induces expression of its targets Fabp6, Shp, Ost $\alpha /$ Ost $\beta$. B, Western diet induces disruption of BA transport in colonocytes after 12 months of feeding. Concentration of DCA and LCA increases in the colon lumen with consumption of high fat, but reduces inside the tissue. BAs are imported predominantly by ASBT, in which downregulation may be caused by increased levels of cholesterol and reduced vitamin D in WD, triggering inactivation of SREBP2 or VDR, respectively. ASBT, FABP6, and OST $\beta$ have reduced abundance, indicating impaired BA transport. As an effect, activity of FXR is reduced, which causes decreased expression of its target genes such as Fabp6, Ost $\alpha / O s t \beta$, and Shp. C, Prolonged feeding with WD (12 to 18 months) increases DCA concentration within the tissue, which increases FXR activity, resulting in many upregulated FXR target genes. At the same time, luminal concentration of DCA and CA reduces. Blue arrows and letters, observed lower abundance or expression; red arrows and letters, higher abundance or expression. Black letters, unchanged expression. Interestingly, mice with normal BA transport, fed with control diet, developed significantly fewer colonic neoplasias, whereas mice fed with WD showed attenuated BA transport at 12 months and later in life (18 and 21 months) developed substantially more colonic neoplasias and tumors. 
FABP6 and other FXR targets, Shp and OST $\beta$, via decreased activity of FXR.

Import rate of BAs into the colonocytes is determined by abundance of ASBT, which transcription is regulated by multiple pathways. Here, downregulated ASBT expression, reduced BA uptake, and increased fecal BAs reassemble observations detected by a study that demonstrates downregulation of ASBT by increased cholesterol via SREBP2 (41). Interestingly, Western diet used in our study was modified by substituting plant-based fat with anhydrous milk fat (Supplementary Table S1), which contains increased amount of cholesterol. Furthermore, inactivation of SREBP2 was identified in systems biology analysis of LC-MS proteomics (Supplementary Fig. S7). In addition, increased cytokines and reduced activity of vitamin $\mathrm{D}$ receptor (VDR) have been shown to reduce transcription of Asbt $(42,43)$ in line with our IPA network analysis that pointed to increased inflammation, previously attributed to WD (4), and decreased content of vitamin D in Western diet (Supplementary Table S1). Taken together, our data strongly suggest that WD most likely modulates ASBT abundance via several mechanisms: increased cholesterol and SREBP2, reduced activity of VDR, and increased cytokines due to inflammation (Fig. $5 \mathrm{~B}$ and $\mathrm{C})$.

Reduced amount of intracellular secondary BAs, DCA especially, downregulates transcriptional activity of FXR. Targeted disruption of $\mathrm{Fxr}$ gene in mice $\left(\mathrm{Fxr}^{-/-}\right)$results in defective feedback inhibition of hepatic BA synthesis and elevated BA excretion in association with loss of FABP6 in the intestine (44), but mice do not develop intestinal tumors. However, FXR deficiency increases intestinal cell proliferation, inflammation, and promotes carcinogenesis in murine models of intestinal cancers $\left(A p c^{\mathrm{Min}}\right.$ mice and azoxymethane-treated mice; ref. 11). Another study shows that not merely the elevation of circulating BA per se but the absence of FXR in the intestinal epithelium increases susceptibility to intestinal tumorigenesis (12). Interestingly, we show that increased proliferation detected in 12-month WD mice coincides with decreased FXR activity, rather than expression (Fig. 5B and C).

It is worth noticing that patients with inflammatory bowel disorder have repressed FXR and SHP in the ileum and colon, and activation of FXR has been shown to reduce intestinal inflammation $(45,46)$. Our findings add-up to already growing evidence that BAs and FXR are important factors in colon inflammation (47) and colorectal cancer etiology, suggesting that intracellular BA concentration may have a greater effect than previously anticipated.

Studies on colon cancer cell lines have shown contradictory effects of BAs on MUC2 expression depending on length of the treatment and concentration of BAs (48) urging in vivo studies to clarify effects of BAs on MUC2, an important tumor-suppressor gene in colorectal cancer (49). Here, we detected an increased expression of MUC2 in 12-month WD, suggesting normal homeostatic response to $\mathrm{WD}$ challenge in order to

\section{References}

1. Wiseman M.The second world cancer research fund/American Institute for Cancer Research expert report. Food, nutrition, physical activity, and the prevention of cancer: A global perspective. Proc Nutr Soc 2008; 67:253-6.

2. Hodge AM, Williamson EJ, Bassett JK, MacInnis RJ, Giles GG, English DR. Dietary and biomarker estimates of fatty acids and risk of colorectal cancer. Int J Cancer 2015;137:1224-34. protect the epithelium. However, longer feeding decreased its expression (Supplementary Fig. S8), suggesting an increased cancer predisposition (50).

In summary, we show that WD increases tumorigenesis by swaying BA homeostasis in colon. Fecal BA concentrations were elevated in WD-fed mice, whereas in corresponding colon tissue, concentrations decreased in association with defective transport, as demonstrated by reduced expressions of BA transporters, FABP6, OST $\beta$, and ASBT. Moreover, decreased intracellular concentrations of secondary BAs, DCA, and LCA indicate reduced activity of FXR. Because AIN mice with active FXR and BA transport develop significantly fewer neoplastic lesions than WD-fed mice, we suggest that active BA transport is necessary for prevention of tumorigenesis. Overall, the results support previously proposed suggestion (51) that FXR agonists could be useful for preventive colorectal cancer treatment in Western populations.

\section{Disclosure of Potential Conflicts of Interest}

No potential conflicts of interest were disclosed.

\section{Authors' Contributions}

Conception and design: D. Dermadi, L. Sarantaus, M. Nyström

Development of methodology: D. Dermadi, S. Valo, R. Soliymani, N. Sipari

Acquisition of data (provided animals, acquired and managed patients, provided facilities, etc.): D. Dermadi, S. Valo, S. Ollila, R. Soliymani, N. Sipari, L. Sarantaus, M. Baumann, M. Nyström

Analysis and interpretation of data (e.g., statistical analysis, biostatistics, computational analysis): D. Dermadi, S. Valo, N. Sipari

Writing, review, and/or revision of the manuscript: D. Dermadi, S. Valo, S. Ollila, R. Soliymani, L. Sarantaus, J. Linden, M. Baumann, M. Nyström

Administrative, technical, or material support (i.e., reporting or organizing data, constructing databases): D. Dermadi, S. Valo, M. Pussila

Study supervision: D. Dermadi, M. Baumann, M. Nyström

Other (histopathologic evaluation of the colon tumors): J. Linden

\section{Acknowledgments}

The authors wish to thank Dr. Marja Mutanen for help with planning the feeding experiments.

\section{Grant Support}

This study was supported by grants from European Research Council (2008-AdG-232635; M. Nyström), Jane and Aatos Erkko Foundation (M. Nyström), The Sigrid Juselius Foundation (M. Nyström), Finnish Cancer Organizations (M. Nyström), Integrative Life Sciences (ILS) Doctoral Program (D. Dermadi and S. Valo), University of Helsinki.

The costs of publication of this article were defrayed in part by the payment of page charges. This article must therefore be hereby marked advertisement in accordance with 18 U.S.C. Section 1734 solely to indicate this fact.

Received October 24, 2016; revised March 8, 2017; accepted April 10, 2017; published OnlineFirst April 17, 2017.

3. Risio M, Lipkin M, Newmark H, Yang K, Rossini FP, Steele VE, et al. Apoptosis, cell replication, and Western-style diet-induced tumorigenesis in mouse colon. Cancer Res 1996;56:4910-6.

4. Erdelyi I, Levenkova N, Lin EY, Pinto JT, Lipkin M, Quimby FW, et al. Western-style diets induce oxidative stress and dysregulate immune responses in the colon in a mouse model of sporadic colon cancer. J Nutr 2009;139:2072-8. 
5. Newmark HL, Yang K, Lipkin M, Kopelovich L, Liu Y, Fan K, et al. A Westernstyle diet induces benign and malignant neoplasms in the colon of normal C57Bl/6 mice. Carcinogenesis 2001;22:1871-5.

6. Đermadi Bebek D, Valo S, Pussila M, Reyhani N, Sarantaus L, Lalowski M, et al. Inherited cancer predisposition sensitizes colonic mucosa to address Western diet effects and putative cancer-predisposing changes on mouse proteome. J Nutr Biochem 2014;25:1-11.

7. Reddy BS, Hanson D, Mangat S, Mathews L, Sbaschnig M, Sharma C, et al Effect of high-fat, high-beef diet and of mode of cooking of beef in the diet on fecal bacterial enzymes and fecal bile acids and neutral sterols. J Nutr 1980;110:1880-7.

8. Bernstein H, Bernstein C, Payne CM, Dvorakova K, Garewal H. Bile acids as carcinogens in human gastrointestinal cancers. Mutat Res 2005;589: $47-65$.

9. Ou J, DeLany JP, Zhang M, Sharma S, O'Keefe SJD. Association between low colonic short-chain fatty acids and high bile acids in high colon cancer risk populations. Nutr Cancer 2012;64:34-40.

10. Takano S, Matsushima M, Ertürk E, Bryan GT. Early induction of rat colonic epithelial ornithine and S-adenosyl-L-methionine decarboxylase activities by N-methyl-N'-nitro-N-nitrosoguanidine or bile salts. Cancer Res 1981; 41:624-8.

11. Maran RRM, Thomas A, Roth M, Sheng Z, Esterly N, Pinson D, et al Farnesoid $\mathrm{X}$ receptor deficiency in mice leads to increased intestinal epithelial cell proliferation and tumor development. J Pharmacol Exp Ther 2009;328:469-77.

12. Modica S, Murzilli S, Salvatore L, Schmidt DR, Moschetta A. Nuclear bile acid receptor FXR protects against intestinal tumorigenesis. Cancer Res 2008;68:9589-94.

13. Ajouz H, Mukherji D, Shamseddine A. Secondary bile acids: An underrecognized cause of colon cancer. World J Surg Oncol 2014;12:164.

14. Reeves PG, Nielsen FH, Fahey GC Jr. AIN-93 purified diets for laboratory rodents: Final report of the American Institute of Nutrition ad hoc writing committee on the reformulation of the AIN-76A rodent diet; 1993. Available from:http://naldc.nal.usda.gov/naldc/catalog.xhtml? id $=43850$

15. Wiśniewski JR, Zougman A, Nagaraj N, Mann M. Universal sample preparation method for proteome analysis. Nat Methods 2009;6:359-62.

16. Hagio $M$, Matsumoto $M$, Fukushima $M$, Hara H, Ishizuka S. Improved analysis of bile acids in tissues and intestinal contents of rats using LC/ESIMS. J Lipid Res 2009;50:173-80.

17. Humbert L, Maubert MA, Wolf C, Duboc H, Mahé M, Farabos D, et al. Bile acid profiling in human biological samples: Comparison of extraction procedures and application to normal and cholestatic patients. J Chromatography B 2012;899:135-45.

18. Jäntti SE, Kivilompolo M, Öhrnberg L, Pietiläinen KH, Nygren H, Ores?ič $\mathrm{M}$, et al. Quantitative profiling of bile acids in blood, adipose tissue, intestine, and gall bladder samples using ultra high performance liquid chromatography-tandem mass spectrometry. Anal Bioanal Chem 2014;406:7799-815.

19. Puppolo M, Varma D, Jansen SA. A review of analytical methods for eicosanoids in brain tissue. J Chromatography B 2014;964:50-64.

20. Lehmann EL, Romano JP. Testing statistical hypotheses. New York, NY: Springer Sci Business Media; 2005.

21. Efron B, Tibshirani R, Storey JD, Tusher V. Empirical bayes analysis of a microarray experiment. J Am Stat Assoc 2001;96:1151-60.

22. Drinkwater NR, Klotz JH. Statistical methods for the analysis of tumor multiplicity data. Cancer Res 1981;41:113-9.

23. Wilkins MR, Appel RD, Williams KL, Hochstrasser DF. Proteome research. Berlin Heidelberg: Springer Science \& Business Media; 2008.

24. Newmark HL, Yang K, Kurihara N, Fan K, Augenlicht LH, Lipkin M Western-style diet-induced colonic tumors and their modulation by calcium and vitamin $\mathrm{D}$ in $\mathrm{C} 57 \mathrm{Bl} / 6$ mice: A preclinical model for human sporadic colon cancer. Carcinogenesis 2009;30:88-92.

25. de Magalhães JP.How ageing processes influence cancer. Nat Rev Cancer 2013;13:357-65.

26. Beyaz S, Mana MD, Roper J, Kedrin D, Saadatpour A, Hong S-J, et al. Highfat diet enhances stemness and tumorigenicity of intestinal progenitors. Nature 2016;531:53-8.

27. Khoo NKH, Hebbar S, Zhao W, Moore SA, Domann FE, Robbins ME. Differential activation of catalase expression and activity by PPAR agonists:
Implications for astrocyte protection in anti-glioma therapy. Redox Biol 2013;1:70-9.

28. Shibanuma $\mathrm{M}$, Inoue $\mathrm{A}$, Ushida $\mathrm{K}$, Uchida $\mathrm{T}$, Ishikawa $\mathrm{F}$, Mori $\mathrm{K}$, et al Importance of mitochondrial dysfunction in oxidative stress response: A comparative study of gene expression profiles. Free Radic Res 2011;45: 672-80.

29. Li Q, Shi R, Wang Y, Niu X. TAGLN suppresses proliferation and invasion, and induces apoptosis of colorectal carcinoma cells. Tumour Biol 2013;34:505-13.

30. Franzon VL, Gibson MA, Hatzinikolas G, Woollatt E, Sutherland GR, Cleary EG. Molecular cloning of a novel human PAPS synthetase which is differentially expressed in metastatic and non-metastatic colon carcinoma cells. Int J Biochem Cell Biol 1999;31:613-26.

31. Van den Abbeele A, De Corte V, Van Impe K, Bruyneel E, Boucherie C, Bracke $\mathrm{M}$, et al. Downregulation of gelsolin family proteins counteracts cancer cell invasion in vitro. Cancer Lett 2007;255:57-70.

32. Zimmerman AW, van Moerkerk HT, Veerkamp JH. Ligand specificity and conformational stability of human fatty acid-binding proteins. Int J Biochem Cell Biol 2001;33:865-76.

33. Hwang ST, Urizar NL, Moore DD, Henning SJ. Bile acids regulate the ontogenic expression of ileal bile acid binding protein in the rat via the farnesoid X receptor. YGAST 2002;122:1483-92.

34. Nakahara M, Furuya N, Takagaki K, Sugaya T, Hirota K, Fukamizu A, et al. Ileal bile acid-binding protein, functionally associated with the farnesoid $\mathrm{X}$ receptor or the ileal bile acid transporter, regulates bile acid activity in the small intestine. J Biol Chem 2005;280:42283-9.

35. Praslickova D, Torchia EC, Sugiyama MG, Magrane EJ, Zwicker BL, Kolodzieyski $\mathrm{L}$, et al. The ileal lipid binding protein is required for efficient absorption and transport of bile acids in the distal portion of the murine small intestine. PLoS ONE 2012;7:e50810.

36. Lee H, Zhang Y, Lee FY, Nelson SF, Gonzalez FJ, Edwards PA. FXR regulates organic solute transporters alpha and beta in the adrenal gland, kidney, and intestine. J Lipid Res 2006;47:201-14.

37. Chen F, Ma L, Dawson PA, Sinal CJ, Sehayek E, Gonzalez FJ, et al. Liver receptor homologue-1 mediates species- and cell line-specific bile aciddependent negative feedback regulation of the apical sodium-dependent bile acid transporter. J Biol Chem 2003;278:19909-16.

38. Neimark E, Chen F, Li X, Shneider BL. Bile acid-induced negative feedback regulation of the human ileal bile acid transporter. Hepatology 2004; 40:149-56.

39. Wang H, Chen J, Hollister K, Sowers LC, Forman BM. Endogenous bile acids are ligands for the nuclear receptor FXR/BAR. Mol Cell 1999;3: 543-53.

40. Makishima M, Okamoto AY, Repa JJ, Tu H, Learned RM, Luk A, et al. Identification of a nuclear receptor for bile acids. Science 1999;284: 1362-5.

41. Thomas C, Landrier J-F, Gaillard D, Grober J, Monnot M-C, Athias A, et al. Cholesterol dependent downregulation of mouse and human apical sodium dependent bile acid transporter (ASBT) gene expression: Molecular mechanism and physiological consequences. Gut 2006;55 1321-31.

42. Chen F, Ma L, Sartor RB, Li F, Xiong H, Sun A-Q, et al. Inflammatorymediated repression of the rat ileal sodium-dependent bile acid transporter by c-fos nuclear translocation. YGAST 2002;123:2005-16.

43. Chen X, Chen F, Liu S, Glaeser H, Dawson PA, Hofmann AF, et al. Transactivation of rat apical sodium-dependent bile acid transporter and increased bile acid transport by 1alpha,25-dihydroxyvitamin D3 via the vitamin D receptor. Mol Pharmacol 2006;69:1913-23.

44. Kok T, Hulzebos CV, Wolters H, Havinga R, Agellon LB, Stellaard F, et al. Enterohepatic circulation of bile salts in farnesoid $\mathrm{X}$ receptor-deficient mice: Efficient intestinal bile salt absorption in the absence of ileal bile acid-binding protein. J Biol Chem 2003;278:41930-7.

45. Gadaleta RM, van Erpecum KJ, Oldenburg B, Willemsen ECL, Renooij W, Murzilli S, et al. Farnesoid X receptor activation inhibits inflammation and preserves the intestinal barrier in inflammatory bowel disease. Gut 2011;60:463-72.

46. Nijmeijer RM, Gadaleta RM, van Mil SWC, van Bodegraven AA, Crusius JBA, Dijkstra G, et al. Farnesoid X receptor (FXR) activation and FXR genetic variation in inflammatory bowel disease. PLoS ONE 2011;6:e23745. 
47. Vavassori $\mathrm{P}$, Mencarelli A, Renga B, Distrutti E, Fiorucci S. The bile acid receptor FXR is a modulator of intestinal innate immunity. J Immunol 2009; 183:6251-61.

48. Shekels LL, Lyftogt CT, Ho SB. Bile acid-induced alterations of mucin production in differentiated human colon cancer cell lines. Int J Biochem Cell Biol 1996;28:193-201.

49. Van der Sluis M, De Koning BAE, De Bruijn ACJM, Velcich A, Meijerink JPP, Van Goudoever JB, et al. Muc2-deficient mice spontaneously develop colitis, indicating that MUC2 is critical for colonic protection. YGAST 2006;131:117-29.

50. Velcich A, Yang W, Heyer J, Fragale A, Nicholas C, Viani S, et al. Colorectal cancer in mice genetically deficient in the mucin Muc2. Science 2002; 295:1726-9.

51. Chatteriee M, Kashfi K. Cell signaling \& molecular targets in cancer. In Chatterjee M, Kashfi K, editors. New York, NY: Springer Science \& Business Media; 2011. 